\title{
一类新型碱激发胶凝材料催化剂的研究进展
}

\author{
张耀君, 杨梦阳, 康乐, 张力, 张科 \\ (西安建筑科技大学 材料与矿资学院，西安 710055)
}

摘 要: 碱激发固体铝硅酸盐胶凝材料是先进无机非金属材料的前瞻性研究领域之一, 本文对碱激发铝硅酸盐胶凝 材料的分类、制备工艺、形成机理以及潜在的应用前景进行了综述; 详尽地论述了碱激发胶凝材料基新型催化剂的 最新研究进展，综合分析了碱激发胶凝材料作为结构材料研究的局限性，展望了该材料作为新型催化材料的发展 动态。

关＼cjkstart键＼cjkstart词：碱激发胶凝材料；固体铝硅酸盐；制备工艺；碱催化机理；胶凝材料催化剂；综述 中图分类号: TQ172 文献标识码: A

\section{Research Progresses of New Type Alkali-activated Cementitious Material Catalyst}

\author{
ZHANG Yao-Jun, YANG Meng-Yang, KANG Le, ZHANG Li, ZHANG Ke
}

(College of Materials and Mineral Resources, Xi' an University of Architecture and Technology, Xi'an 710055, China)

\begin{abstract}
Alkali-activated solid aluminosilicate-based cementitious material is one of prospective research fields of advanced inorganic non-metallic materials. Its classification, preparation process, formation mechanism, and potential applications are reviewed in this paper. It is considered that its microstructure and chemical characteristics intensively depend on the raw materials and synthesis conditions. Geopolymers derive from alkali-activated metakaolin or fly ash with low calcium content, while the amorphous calcium silicate hydrate (C-S-H) gels root in the chemical-activated solid wastes of granular blast furnace slag, steel slag and other solid aluminosilicate wastes with high calcium contents. Even though durability of alkali-activated cementitious materials as the building structure materials has been widely studied in the past decades, the intrinsic brittleness still restricts their applications in the field of civil and building engineering. Therefore, exploration of a new applied approach is by far the best option. In recent years, many researches report that the alkali-activated cementitious materials are used as novel precursors and catalysts for some kinds of heterogeneous reactions. The latest research progresses on alkali-activated cementitious material-based catalysts are discussed.
\end{abstract}

Key words: alkali-activated cementitious material; solid aluminosilicate; preparation technics; alkali-catalytic mechanism; cementitious material catalyst; review

碱激发固体铝硅酸盐胶凝材料是先进无机非金 属材料的前瞻性研究领域之一，以工业固体废弃物 (粉煤灰、矿渣、钢渣、煤矸石及各种尾矿等)为原 料，与碱性激发剂溶液反应制备碱激发铝硅酸盐胶
凝材料具有工艺简单、无需烧制、成本低廉、能耗 低、 $\mathrm{CO}_{2}$ 印迹少，保持生态平衡、环境友好和可实 现固体废弃物规模化资源循环利用等优点，是一类 蕴藏巨大发展潜力的先进绿色材料。在数十年的发

收稿日期: 2015-08-31；收到修改稿日期：2015-10-12

基金项目：国家自然科学基金(21346011); 陕西省自然科学基金重点项目(2013JZ014)

National Natural Science Foundation of China (21346011); Natural Science Basic Research Plan of Shaanxi Province (2013JZ014)

作者简介: 张耀君(1959-)，男，教授. E-mail: zhangyaojun@xauat.edu.cn 
展历程中, 取得了制备工艺、影响因素、水化机理、 工作性能、耐久性能等大量研究成果, 许多研究者 对碱激发胶凝材料的研究进展进行了较系统的综 述 $^{[1-5]}$, 但其作为催化剂的研究进展尚未见相关综 述报道。由于各种固废的产地、化学组成以及矿物 相不尽相同, 制备工艺及碱激发胶凝材料的性能千 差万别, 难以形成普适性的行业标准或技术操作规 范, 制约了其作为建筑结构材料的规模化、商业化 推广应用进程 ${ }^{[5-6]}$ 。欲突破目前研究的瓶颈, 开辟碱 激发胶凝材料作为非结构材料应用的新途径, 特别 作为一类新型的高活性、长寿命、低成本的催化材 料, 为其创造新的价值空间, 是该材料发展的驱动 力和出路所在。

\section{1 碱激发胶凝材料的分类及制备工艺}

\section{1 碱激发胶凝材料的分类}

碱激发胶凝材料的研究始于上世纪 40 年代, 经 历了漫长的发展历程。碱激发胶凝材料按其原料的 组成以及化学结构可分为两类, 一类是以上世纪 50 年代前苏联的 Glukhovsky 以及上世纪 70 年代法国 的 Davidovits 为代表研究的碱激发固体无钲或低钙 铝硅酸盐胶凝材料, 该类材料是以固体无钙或低钙 铝硅酸盐为原料, 与碱性激发剂反应, 生成由 $\left[\mathrm{SiO}_{4}\right]^{4+}$ 四面体和 $\left[\mathrm{AlO}_{4}\right]^{5+}$ 四面体通过桥氧键连接的 类沸石结构的水硬性胶凝材料, 其凝胶体系可简写 为 $\mathrm{R}_{2} \mathrm{O}-\mathrm{Al}_{2} \mathrm{O}_{3}-\mathrm{SiO}_{2}-\mathrm{H}_{2} \mathrm{O}(\mathrm{R}=\mathrm{Na}, \mathrm{K})$; Glukhovsky 等 ${ }^{[7]}$ 将其称为土壤硅酸盐(soil silicates), Davidovits ${ }^{[8-9]}$ 将 其称为地质聚合物 ( geopolymer), 后来的一些学者 将其称为土聚材料(earth materials)或土聚水泥(soil cements) ${ }^{[10]}$ 、碱键合陶瓷(alkali-bounded-ceramics) ${ }^{[11]}$ 、 低温铝硅酸盐玻璃 (low-temperature aluminosilicate glasses) ${ }^{[12]}$ 和无机聚合物(inorganic polymer) ${ }^{[7]}$ 等。尽 管不同研究者给出了不同的命名, 但碱激发缩聚反 应是该类材料的本质所在。另一类是 20 世纪 40 年 代以 Purdon ${ }^{[13]}$ 为代表研究的碱激发固体高钙铝硅 酸盐胶凝材料, 是化学激发剂与固体高钙铝硅酸盐 原料反应形成的一类具有无定形的水化硅酸钻 $(\mathrm{CSH})$ 结构的水硬性胶凝材料, 其凝胶体系可简化 为 $\mathrm{R}_{2} \mathrm{O}-\mathrm{CaO}-\mathrm{Al}_{2} \mathrm{O}_{3}-\mathrm{SiO}_{2}-\mathrm{H}_{2} \mathrm{O}(\mathrm{R}=\mathrm{Na}, \mathrm{K})$ 。

\section{2 碱激发胶凝材料的制备工艺}

无钙或低钙碱激发胶凝材料的制备是以天然铝 硅酸盐矿物或无钻或低钙的工业固体废弃物(如: 高岭土、天然火山灰、天然沸石、低钻粉煤灰)为原 料; 而高钙碱激发胶凝材料的制备是以含高钙的工
业固体废弃物(如: 矿渣、钢渣、赤泥、镁渣、各种 尾矿)为原料。通常以固体 $\mathrm{NaOH} 、 \mathrm{KOH} 、 \mathrm{Na}_{2} \mathrm{SiO}_{3}$ 或水玻璃等为激发剂, 将一定水灰比、一定浓度的 碱激发剂水溶液, 在摚拌条件下倒入盛有固体粉体 原料的容器中形成浆体, 再将浆体倾入模具中进行 成型养护。可根据原料的属性, 选取不同的养护制 度。无钙或低钙原料(如: 高岭土、粉煤灰)由于其活 性低, 一般选择 $40 \sim 100^{\circ} \mathrm{C}$ 的热养护制度 ${ }^{[14-17]}$, 对于 高钙原料(如: 矿渣) 室温养护即可 ${ }^{[18-21]}$, 检测试件 不同龄期的力学性能。

在无钻或低钻碱激发胶凝材料的制备过程中, 一旦固体铝硅酸盐粉体原料与激发剂的水溶液混合, 随着养护龄期的延长, 碱激发胶凝材料形成更密实 的多晶或半晶体的类沸石结构, 但比沸石具有更高 的力学性能, 通常被称为碱激发地质聚合物胶凝材 料。在高钻碱激发胶凝材料的制备过程中, 当固体 高钙铝硅酸盐原料与碱激发剂的水溶液混合, 室温 养护下就能快速水化硬化, 转化成无定形的水化硅 酸钙(CSH)块体材料, 通常被称为碱激发胶凝材料。 在制备过程中，原料的氧化物及矿物相组成、粒度 分布、激发剂种类及浓度、 $\mathrm{pH} 、 \mathrm{Si} / \mathrm{Al}$ 比、 $\mathrm{Ca} / \mathrm{Si}$ 比、 水灰比、养护温度等直接影响着碱激发胶凝材料的 矿物组成、力学性能、孔结构以及耐久性能等。

$\mathrm{Xu}$ 等 ${ }^{[22]}$ 采用 16 种天然 $\mathrm{Si}-\mathrm{Al}$ 矿物分别与高岭 土混合, 合成具有一定抗压强度的碱激发地质聚合 物胶凝材料。研究发现若仅以天然 Si-Al 矿物为原 料, 生成的碱激发胶凝材料容易开裂; 但如果仅以 高岭土为原料, 而缺少其它铝硅酸盐矿物, 生成的 碱激发地质聚合物表现出低的抗压强度, 因此, 碱 激发高岭土生成的地质聚合物与其它铝硅酸盐矿物 形成的胶凝材料之间存在着很强的协同作用 ${ }^{[15]}$ 。

由于采用天然矿物制备的碱激发地质聚合物的 抗压强度较低, 后来发展为天然矿物与固体废弃物 为混合原料的碱激发胶凝材料, 最具代表性的一类 是以偏高岭土与粒化高炉矿渣为混合原料, 另一类 是以粉煤灰与粒化高炉矿渣为混合原料。碱激发偏 高岭土一矿渣基胶凝材料是由碱激发偏高岭土生成 的地质聚合物凝胶与碱激发矿渣生成的水化硅酸钙 凝胶二种分立的凝胶体系所组成，由于两种凝胶表 面的相互作用, 亦存在相互交织的混合相, 块体材 料显示出高的力学性能 ${ }^{[23-27]}$ 。Yip 等 ${ }^{[28-30]}$ 在碱激发 粉煤灰一矿渣基胶凝材料的研究中发现, CSH 凝胶 与地质聚合物凝胶共存, 表明两种凝胶具有相容性, 这就意味着一种新的结构材料的诞生。除此之外, 人们亦尝试采用其它各种固体铝硅酸盐原料制备碱 
激发胶凝材料，其中包括碱激发电弧铁一镍渣基胶 凝材料 ${ }^{[31]}$, 碱激发矿渣一赤泥基胶凝材料 ${ }^{[32]}$, 碱激 发铇矿尾矿基胶凝材料 ${ }^{[33]}$, 碱激发废弃流化床催化 裂化催化剂基胶凝材料 ${ }^{[34]}$ 等。

\section{2 碱激发胶凝材料的形成机理}

\section{1 无(低)钙铝硅酸盐胶凝材料的形成机理}

碱激发固体无(低)钙铝硅酸盐胶凝材料的形成 机理包括 Glukhovsky 形成机理以及 Davidovits 形 成机理。图 1 是 Glukhovsky 在 20 世纪 50 年代提 出的碱激发固体无(低)钻铝硅酸盐地质聚合胶凝 材料的形成机理 ${ }^{[7]}$ 。他把固体无(低)钙铝硅酸盐地 质聚合胶凝材料的形成机理分为三个阶段(如图 1 所示): (1)在碱激发剂的作用下，固体铝硅酸盐颗粒 表面开始溶解, 活性铝酸盐和硅酸盐物种溶出, 这 些复杂的物种形成一种过饱和的铝硅酸盐溶液, 物 种之间通过脱水反应在水相中形成寡聚物(凝结), 称为解体一凝结 (destruction-coagulation); (2)在水相 中的寡聚物经过进一步的缩聚反应形成大的网络结 构的凝胶 (gel 1) 称为凝结-缩聚 (coagulation- condensation); (3)缩聚产物经继续脱水反应再组织形成凝 胶( gel 2), 该凝胶进一步发生重组, 凝胶网络结构
的连通性增加; 胶体生长, 成核达到某一临界尺寸, 生成半晶体类沸石结构的地质聚合物, 称为缩聚晶化(condensation crystallization)。在这三个阶段中, 固体铝硅酸盐原料转化为碱性铝硅酸盐溶液是关 键阶段。缩聚反应失去的水是固体铝硅酸盐颗粒在 溶解过程中所消耗的水, 因此, 水充当了一种反应 介质，经缩聚反应脱去的水残留于凝胶的孔道中， 这种胶体结构通常被称为含有铝硅酸盐凝胶和水 的二相体系。

Davidovits 提出碱激发固体无(低)钙铝硅酸盐 地质聚合物是由 $\left[\mathrm{SiO}_{4}\right]^{4+}$ 四面体和 $\left[\mathrm{AlO}_{4}\right]^{5+}$ 四面体通 过共享氧原子交替结合形成的-Si-O-Al-的骨架结 构, $\mathrm{Na}^{+}$或 $\mathrm{K}^{+}$碱金属阳离子嵌入骨架的空穴中，以平 衡 $\mathrm{Al}^{3+}$ 的负电荷。根据碱激发地质聚合物中 $\mathrm{Si}$ 与 $\mathrm{Al}$ 的比值将其分为三类 ${ }^{[8-9]}$ 。当固体硅铝酸盐原料中的 $\mathrm{Si}: \mathrm{Al}=1$ 时, 在 $\mathrm{NaOH}$ 或 $\mathrm{KOH}$ 激发剂水溶液中固 体硅铝酸盐原料溶出, 生成正硅铝酸钠盐或正硅铝 酸钾盐，如方程式(1)所示，该钠盐或钾盐在碱性条 件下进一步发生缩聚反应,生成聚硅铝酸盐 Poly- sialate (PS)型的骨架结构, 如方程式(2)所示。当固体 硅铝酸盐原料中的 $\mathrm{Si}: \mathrm{Al}=2$ 时, 可获得 Poly-sialatesiloxo (PSS)型的骨架结构, 如方程式(3)和(4)所 示。当固体硅铝酸盐原料中的 $\mathrm{Si}: \mathrm{Al}=3$ 时, 可

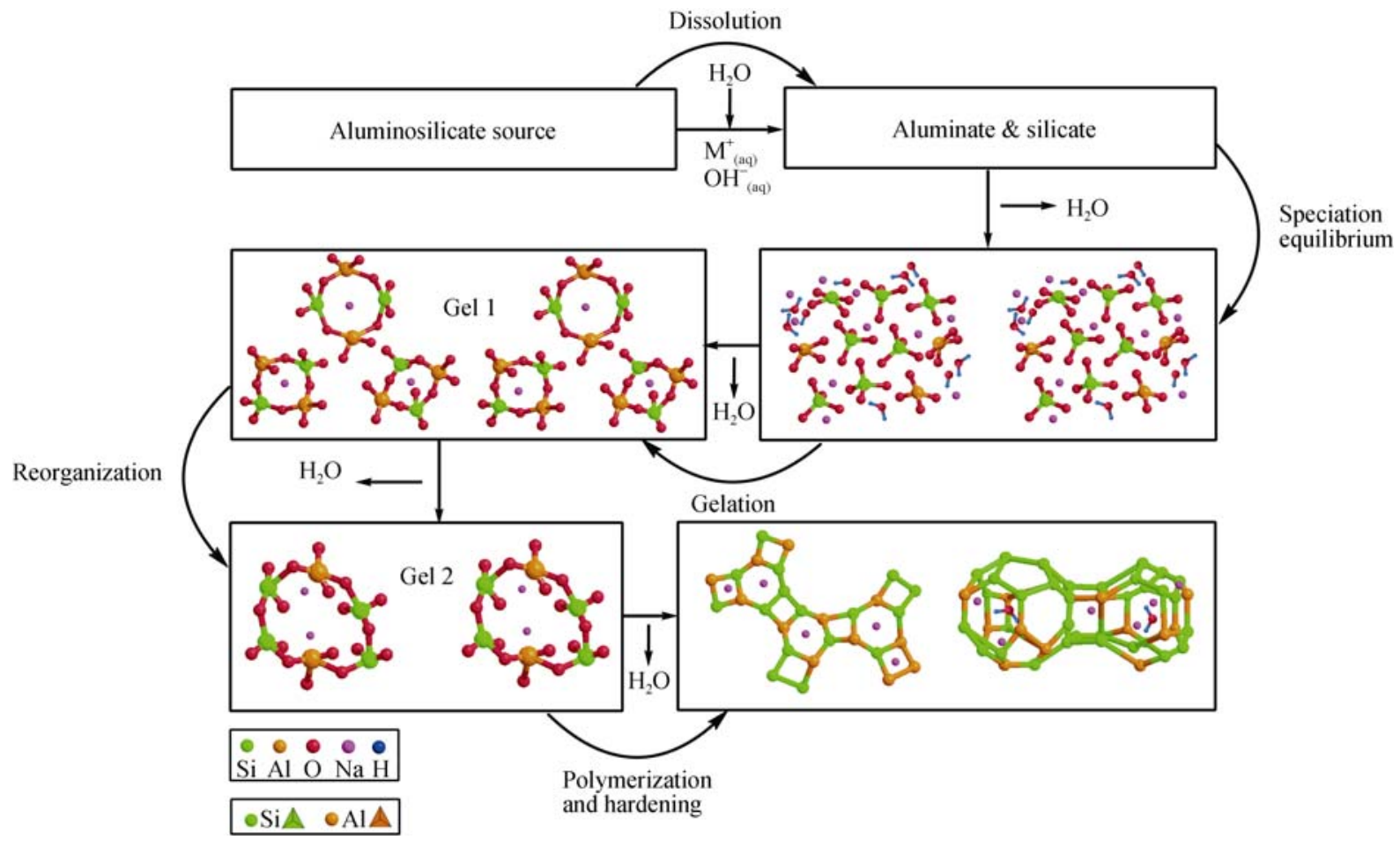

图 1 碱激发固体铝硅酸盐地质聚合物的形成机理 ${ }^{[7]}$

Fig. 1 Formation mechanism of alkali-activated solid aluminosilicate-based geopolymer ${ }^{[7]}$ 
获得 Poly-sialate-disiloxo (PSDS)型的骨架结构, 如方 程式(5)和(6)所示。碱激发地质聚合物是由链状、片 状或三维网络结构的 $\left[\mathrm{SiO}_{4}\right]^{4+}$ 和 $\left[\mathrm{AlO}_{4}\right]^{5+}$ 四面体相互 结合的各种单元所组成 ${ }^{[35]}$ 。碱激发地质聚合物的形 成是一个放热反应，在激发剂溶液中，固体铝硅酸 盐溶解形成低聚物(如: 二聚或三聚体等)或原硅酸或 原铝酸单体，这些低聚物或单体从固体原料的表面
扩散进入溶液，在溶液中相互之间发生缩聚反应形 成链状、片状或者三维网络结构的溶胶，最终硬化形 成凝胶。Davidovits 等 ${ }^{[3,8-9]}$ 认为, 激发剂中 $\mathrm{OH}^{-}$离子 的浓度越高，越有利于不同的硅酸盐物种及铝酸盐 物种的溶解, 从而加速缩聚反应进程; 但是, 如果在 过高的碱性环境下(整个体系中 $\mathrm{Na}_{2} \mathrm{O}>30 \mathrm{~mol} \%$ ), 硅 酸根阴离子的连通性就会降低, 对缩聚反应不利 ${ }^{[35]}$ 。

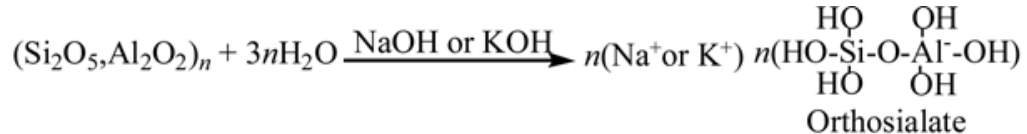

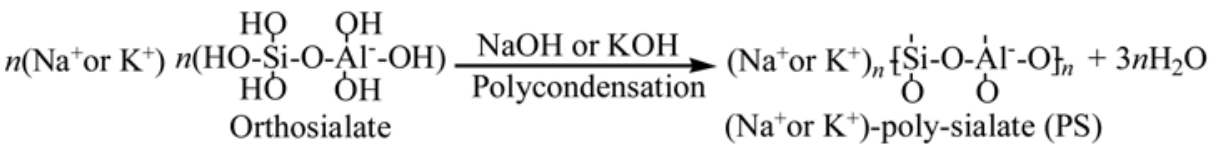

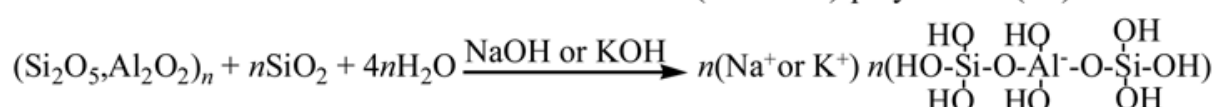

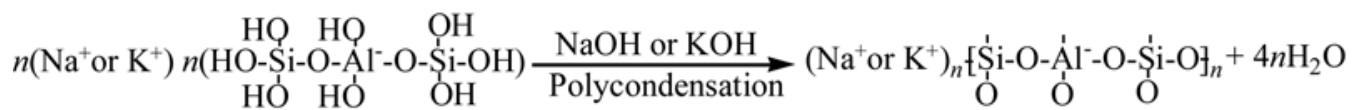

$$
\begin{aligned}
& \left(\mathrm{Na}^{+} \text {or } \mathrm{K}^{+}\right) \text {-poly-sialate-siloxo (PSS) }
\end{aligned}
$$

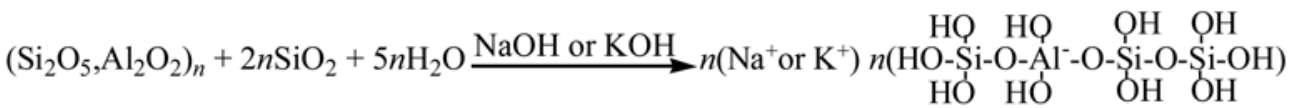

$$
\begin{aligned}
& \mathrm{HO} \quad \mathrm{HO} \quad \mathrm{OH} \quad \mathrm{OH}
\end{aligned}
$$

\section{2 高钙铝硅酸盐胶凝材料的碱催化机理}

Purdon ${ }^{[13]}$ 提出了碱激发固体高钲铝硅酸盐基胶 凝材料的碱催化机理, 如方程式(7) (10)所示。在激 发剂 $\mathrm{NaOH}$ 的作用下, 固体高钙铝硅酸盐溶解生成 $\mathrm{Si}(\mathrm{OH})_{4} 、 \mathrm{Al}(\mathrm{OH})_{3} 、 \mathrm{Ca}(\mathrm{OH})_{2}$ 等, $\mathrm{Si}(\mathrm{OH})_{4}$ 与激发剂反 应生成 $\mathrm{Na}_{2} \mathrm{SiO}_{3}$, 如方程式(7) 所示; $\mathrm{Na}_{2} \mathrm{SiO}_{3}$ 、 $\mathrm{Ca}(\mathrm{OH})_{2}$ 以及 $\mathrm{H}_{2} \mathrm{O}$ 三者反应生成水化硅酸钻 $(\mathrm{CSH})$ 凝胶和 $\mathrm{NaOH}$, 如方程式(8)所示。固体高钙铝硅酸 盐溶出的 $\mathrm{Al}(\mathrm{OH})_{3}$ 与激发剂 $\mathrm{NaOH}$ 反应生成 $\mathrm{NaAlO}_{2}$,

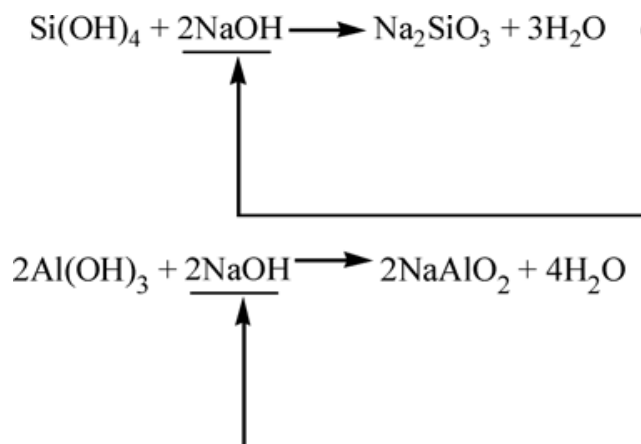

\section{$\left(\mathrm{Na}^{+}\right.$or $\left.\mathrm{K}^{+}\right)$-poly-sialate-disiloxo (PSDS)}

如方程式(9)所示; 生成的 $\mathrm{NaAlO}_{2} 、 \mathrm{Ca}(\mathrm{OH})_{2}$ 以及 $\mathrm{H}_{2} \mathrm{O}$ 反应生成水化铝酸钙 $(\mathrm{CAH})$ 凝胶和 $\mathrm{NaOH}$, 如 方程式(10)所示。对于实际上极其复杂的碱激发反 应，采用简单的方程式示意性的表明碱激发剂 $\mathrm{NaOH}$ 没有参与反应，而是扮演着一种催化剂的角 色, 循环催化 $\mathrm{Si}(\mathrm{OH})_{4}$ 反应生成 $\mathrm{CSH}$; 循环催化 $\mathrm{Al}(\mathrm{OH})_{3}$ 反应生成 $\mathrm{CAH}$ 。在激发剂的催化下，固体 高钻铝硅酸盐不断溶解，水化硬化生成无定形 CSH 和 $\mathrm{CAH}$ 胶凝材料。

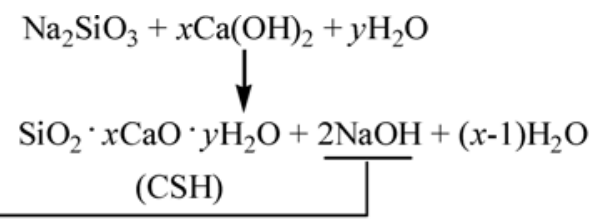

$$
\begin{aligned}
& 2 \mathrm{NaAlO}_{2}+\underset{\mathrm{pCa}(\mathrm{OH})_{2}}{2 \mathrm{qH}_{2} \mathrm{O}} \\
& \mathrm{Al}_{2} \mathrm{O}_{3} \cdot \mathrm{pCaO} \cdot \mathrm{qH}_{2} \mathrm{O}+\underset{\mathrm{CAH}}{\mathrm{NaOH}} \\
& (\mathrm{CH})
\end{aligned}
$$

碱激发固体铝硅酸盐胶凝材料具有可调的初凝 及终凝, 低收缩率及低的热导系数 ${ }^{[36]}$, 耐磨 ${ }^{[37]}$, 耐 高温 ${ }^{[38]}$, 优良的粘结性能 ${ }^{[39]}$, 低的碱集料反应 ${ }^{[40]}$, 抗酸腐蚀 ${ }^{[41-42]}$, 抗硫酸盐腐蚀 ${ }^{[43]}$, 耐海水侵蚀 ${ }^{[44]}$, 抗 氯离子侵蚀 ${ }^{[45]}$, 抗冻融 ${ }^{[46]}$ 等特性, 使其可作为潜在
的结构材料用于铺设路面、现浇混凝土或预制混凝 土、建筑用楼板、油井水泥、密封材料、防水材料, 路面修补材料等建筑材料领域。同时，碱激发胶凝 材料是一类结构致密的孔性材料, 作为非建筑材料

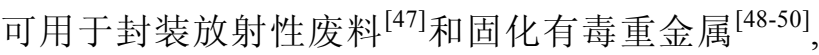


但值得注意的是并非每种碱激发胶凝材料都具备上 述所有特性。

\section{3 新型碱激发胶凝材料催化剂}

\section{1 碱激发偏高岭土基胶凝材料催化剂}

Sazama 等 $^{[51]}$ 报导了过渡金属或贵金属负载的 偏高岭土基以及偏高岭土一矿渣基胶凝材料催化剂, 并将其应用于挥发烃癸烷的完全氧化反应，以及氨 对 $\mathrm{NO}_{x}$ 的催化还原反应。完全氧化反应是模拟贫氧 (低于空气中 $\mathrm{O}_{2}$ 浓度)下的癸烷燃烧排放过程, 在反 应器入口处, 采用 $6 \% \mathrm{O}_{2} / \mathrm{He}$ 混合气将 $250 \times 10^{-6}$ 的正 癸烷蒸汽带入固定床反应装置中，空速(GHSV) $60000 \mathrm{~h}^{-1}$, 催化剂 $100 \mathrm{mg}$, 采用气相色谱检测。癸 烷在 $\mathrm{PtNH}_{4}$-Geo-2、FeKCa-Geo-2 和 $\mathrm{CoNH}_{4}$-Geo-2 催化剂上的完全氧化反应转化率以及 $\mathrm{CO}_{2}$ 和 $\mathrm{CO}$ 的 选择性如图 2(a)和(b)所示。从图 2(a)可见, 癸烷在 $\mathrm{PtNH}_{4}-\mathrm{Geo}-2$ 催化下, 低温氧化具有最高的转化率; 在反应温度 $100 \sim 200^{\circ} \mathrm{C}$ 范围内, 癸烷的转化率达 $98 \%$ 以上, 并几乎完全转换为 $\mathrm{CO}_{2}$, 如图 2(b) 所示。而 FeKCa-Geo-2 和 $\mathrm{CoNH}_{4}$-Geo-2 催化癸烷氧化反应大 约在 $120 \sim 480^{\circ} \mathrm{C}$ 较高的温度范围。从图 2(b)可以看 出, 随着反应温度的升高, $\mathrm{CO}_{2}$ 的选择性逐渐增强, 而 $\mathrm{CO}$ 的选择性减弱。

Gasca-Tirado 等 ${ }^{[52-53]}$ 制备了 Na-型碱激发偏高岭土基 胶凝材料, 经过 $\mathrm{NH}_{4}{ }^{+}$交换, 再与 $\left(\mathrm{NH}_{4}\right)_{2} \mathrm{TiO}\left(\mathrm{C}_{2} \mathrm{O}_{4}\right)_{2} \cdot \mathrm{H}_{2} \mathrm{O}$ 水溶液进行离子交换, 或直接用 $\mathrm{Na}$ - 型胶凝材料与 $\left(\mathrm{NH}_{4}\right)_{2} \mathrm{TiO}\left(\mathrm{C}_{2} \mathrm{O}_{4}\right)_{2} \cdot \mathrm{H}_{2} \mathrm{O}$ 水溶液进行离子交换, 制得 Ti-型偏高岭土基胶凝材料催化剂, 该催化剂对亚甲
基蓝染料以及 2-丁酮具有一定的光催化降解活性。 Candamano 等 ${ }^{[54]}$ 制备了负载 $\mathrm{NiO}$ 的碱激发偏高岭土 基催化剂，评价了该催化剂用于乙醇蒸汽重整反应 制氢以及乙醇部分氧化制合成气的催化反应，在乙 醇重整反应中，当反应温度 $700^{\circ} \mathrm{C}$ 时，乙醇发生完全 转换, 可得到 $70 \mathrm{~mol} \%$ 的 $\mathrm{H}_{2}$ 。

Sharma 等 ${ }^{[55]}$ 将负载 $\mathrm{CaO}$ 的碱激发偏高岭土基 胶凝材料催化剂用于大豆油经酯交换反应制备生物 柴油研究，其制备工艺是将定量的 $\mathrm{SiO}_{2}$ 溶于 $\mathrm{KOH}$ 溶液, 将偏高岭土缓慢地加入该溶液中形成可流 动的液体称之为地质聚合物树脂，将菜籽油与树 脂 1 : 1 混合形成粘稠状混合物, $60^{\circ} \mathrm{C}$ 养护 $24 \mathrm{~h}$,得到 块体材料，用丁醇反复洗涤除去有机成分，获得孔 性地质聚合物, 经浸渍获得负载 $\mathrm{CaO}$ 的催化剂。将 $100 \mathrm{mg}$ 的催化剂与 $6 \mathrm{~g}$ 无水甲醇加入圆底烧瓶, $67^{\circ} \mathrm{C}$ 搅拌回流，加入 $1 \mathrm{~g}$ 大豆油反应 $2 \mathrm{~h}$, 冷却后加入己 烷进行混合, 离心分离。已烷的上层液中含有生物 柴油和未反应的大豆油，下层液中含有甲醇和丙三 醇, 将分离的上层液 $70^{\circ} \mathrm{C}$ 蒸发除去己烷，获得生物 柴油。

\section{2 碱激发钢渣基胶凝材料催化剂}

Zhang 等 ${ }^{[56]}$ 将 $\mathrm{Ni}$-型碱激发钢渣基胶凝材料催 化剂应用于光催化降解染料研究。从图 3 可见, Na型以及 $\mathrm{Ni}$-型碱激发胶凝材料催化剂对亚甲基蓝染 料的光催化降解率分别为 $53.27 \%$ 和 $94.39 \%$ 。 Ni-型碱 激发胶凝材料催化剂对亚甲基蓝染料高的光催化降 解率活性归因于 $\mathrm{Ni}^{2+}$ 离子与碱激发钢渣基胶凝材料 中含有的方铁矿 $(\mathrm{FeO}) 、$ 碱激发过程中生成的 $\mathrm{Ca}_{2} \mathrm{Fe}_{2} \mathrm{O}_{5}$ 以及胶凝材料的网络结构之间产生强的协同作用的
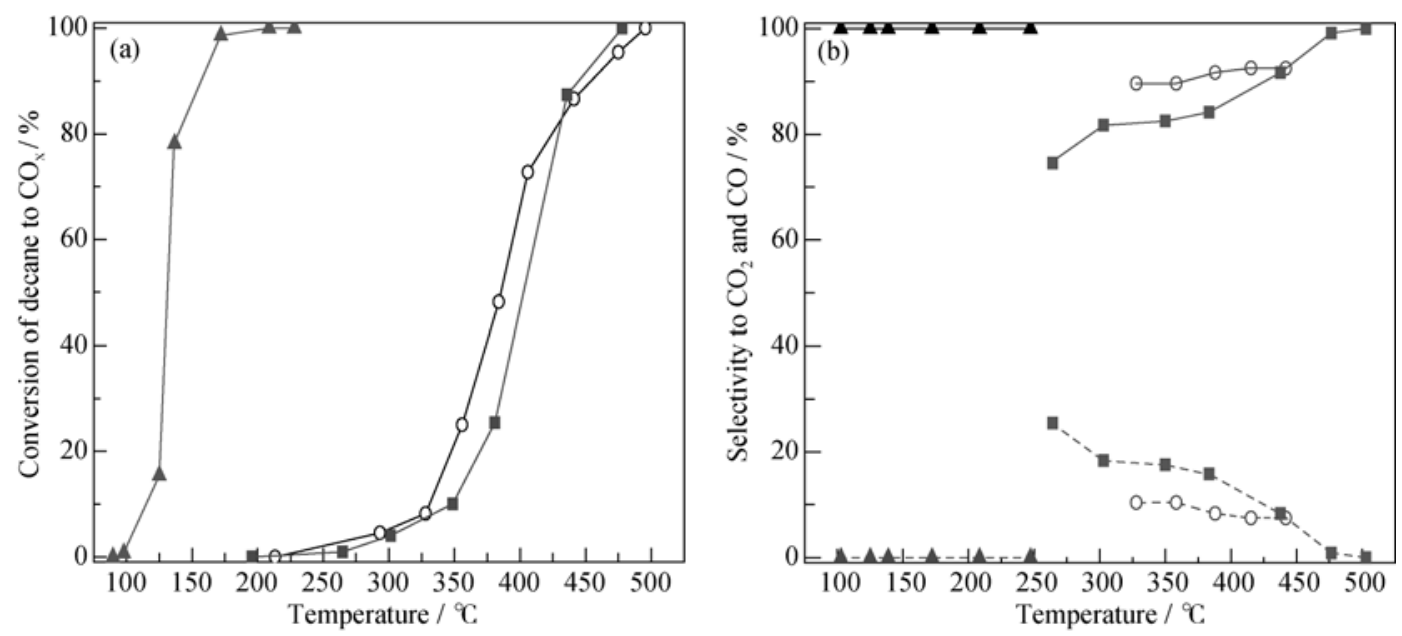

图 2 癸烷在 $\mathrm{PtNH}_{4}-\mathrm{Geo}-2(\boldsymbol{\Delta}) 、 \mathrm{FeKCa}-\mathrm{Geo}-2(\mathrm{O})$ 和 $\mathrm{CoNH}_{4}-\mathrm{Geo}-2$ (听催化剂上的转化率 $(\mathrm{a})$ 以及 $\mathrm{CO}_{2}$ (实线)和 $\mathrm{CO}$ (点线) 的选择性(b) ${ }^{[51]}$

Fig. 2 Conversion of decane (a) and selectivity for $\mathrm{CO}_{2}$ (solid line) and $\mathrm{CO}$ (dot line) (b) over PtNH $\mathrm{H}_{4}-\mathrm{Geo}^{2}$ ( $(\boldsymbol{\Delta})$, FeKCa-Geo-2 (o) and $\mathrm{CoNH}_{4}-\mathrm{Geo}-2(\mathbf{\square})^{[51]}$ 


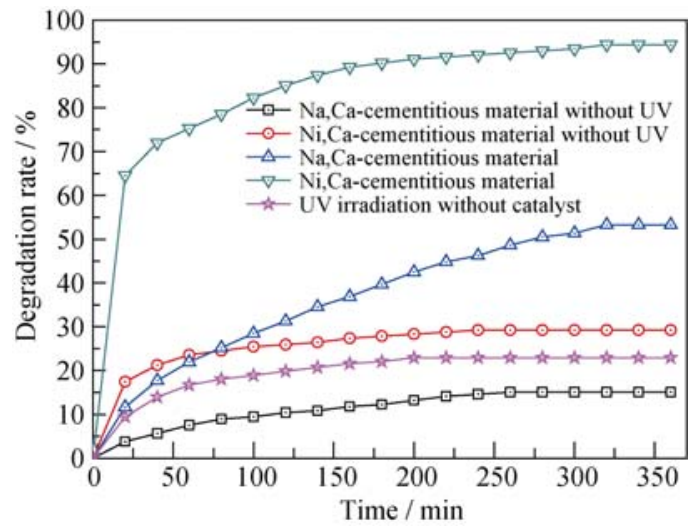

图 3 碱激发钢渣基胶凝材料催化剂对亚甲基蓝染料光催化 降解率 [56]

Fig. 3 Photocatalytic degradation rate of methylene blue dye over alkali-activated steel slag-based gel catalysts ${ }^{[56]}$

结果。Kang 等 ${ }^{[57]}$ 采用孔形成剂合成了孔结构可控 的碱激发钢渣基胶凝材料, 结果表明, 孔形成剂能 够控制介孔的形成, 催化剂介孔孔容的增加, 能显 著地提高光催化孔雀石绿染料的降解率。

\section{3 碱激发粉煤灰基胶凝材料催化剂}

碱激发粉煤灰基胶凝材料可作为除去 $\mathrm{Pb}^{2+}$ 、 $\mathrm{Cu}^{2+} 、 \mathrm{NO}_{x}$ 、磷酸盐、喼化物、氟化物, 放射性 ${ }^{137} \mathrm{Cs}$, ${ }^{90} \mathrm{Sr}$ 、染料分子以及甲醛的吸附剂 ${ }^{[58-62]}$, 碱激发偏高 岭土基胶凝材料能除去水中的 $\mathrm{Pb}^{2+} 、 \mathrm{Cu}^{2+} 、 \mathrm{Cr}^{3+}$ 和 $\mathrm{Cd}^{2+[63]}$ 。碱激发胶凝材料作为吸附剂虽能有效地去 除水中的污染物分子, 但当吸附达到饱和时, 需通 过加热等方式除去吸附质分子, 这就需要增加设备 投入，造成能耗高并产生二次污染等问题。

Zhang 等 ${ }^{[64]}$ 将碱激发粉煤灰基胶凝材料催化剂 用于光催化染料降解研究, 没有催化剂时, 亚甲基 蓝染料的光解率为 $22.97 \%$; 染料在碱激发粉煤灰基 胶凝材料催化剂上的吸附率为 $89.15 \%$, 降解率达到 $92.79 \%$, 最高的降解率源于碱激发粉煤灰基胶凝材 料的网络结构对染料分子的吸附, 以及胶凝材料中 的过渡金属氧化物半导体对染料分子的光催化降解 作用。

\section{4 碱激发矿渣基胶凝材料催化剂}

Zhang 等 ${ }^{[65-66]}$ 报导了采用碱激发缩聚、离子交换 以及浸渍三步反应制备负载 $\mathrm{Fe}_{2} \mathrm{O}_{3}$ 的碱激发矿渣基催 化剂, 实验采用的 Na-ASCM 试块的抗压强度 $86 \mathrm{MPa}$, 经破碎篎分造粒，离子交换后获得 $\mathrm{NH}_{4}-\mathrm{ASCM}$ 载体; 经浸渍负载, 分别制备 $0.5 \mathrm{Fe}_{2} \mathrm{O}_{3} / \mathrm{ASCM} 、 5 \mathrm{Fe}_{2} \mathrm{O}_{3} / \mathrm{ASCM}$ 和 $10 \mathrm{Fe}_{2} \mathrm{O}_{3} / \mathrm{ASCM}$ 催化剂, 用于刚果红染料的光催 化降解评价研究。刚果红染料在催化剂上的吸附 率随 $\mathrm{Fe}_{2} \mathrm{O}_{3}$ 负载量的增加而增加, 分别为: $46.5 \%$ $\left(10 \mathrm{Fe}_{2} \mathrm{O}_{3} / \mathrm{ASCM}\right)>42.5 \%\left(5 \mathrm{Fe}_{2} \mathrm{O}_{3} / \mathrm{ASCM}\right)>21.0 \%\left(0.5 \mathrm{Fe}_{2} \mathrm{O}_{3} /\right.$
$\mathrm{ASCM}$ ), 表明 $\mathrm{Fe}_{2} \mathrm{O}_{3}$ 是刚果红染料吸附的活性中心。 光催化降解活性顺序： $100 \%\left(5 \mathrm{Fe}_{2} \mathrm{O}_{3} / \mathrm{ASCM}\right)>97.3 \%$ $\left(10 \mathrm{Fe}_{2} \mathrm{O}_{3} / \mathrm{ASCM}\right)>93.2 \%\left(0.5 \mathrm{Fe}_{2} \mathrm{O}_{3} / \mathrm{ASCM}\right)>86 \%(\mathrm{Na}-$ $\mathrm{ASCM}$ )。 $5 \mathrm{Fe}_{2} \mathrm{O}_{3} / \mathrm{ASCM}$ 的降解活性最高归因于 $\mathrm{Fe}_{2} \mathrm{O}_{3}$ 纳米粒子均匀地分散于载体表面，提供活性位，从而 促进光催化降解反应。

\section{5 碱激发镁渣基胶凝材料催化剂}

Zhang 等 ${ }^{[67-68]}$ 制备了共负载 $\mathrm{NiO}$ 和 $\mathrm{CuO}$ 的碱激 发镁渣基胶凝材料催化剂, 并将其应用于光催化分 解水制氢以及靛蓝染料降解研究。实验采用的 Na型碱激发镁渣基块体胶凝材料(Na-AMSNC)的抗压 强度 $12.8 \mathrm{MPa}$, 造粒后进行 $\mathrm{NH}_{4}{ }^{+}$离子交换, 得到 $\mathrm{NH}_{4}-\mathrm{AMSNC}$ 催化剂载体; 经共负载 $0.5 \mathrm{wt} \% \mathrm{NiO}$ 和 $0.5 \mathrm{wt} \% \mathrm{CuO}, 400^{\circ} \mathrm{C}$ 焙烧后命名为 $1(\mathrm{NiO}+\mathrm{CuO}) /$ AMSNC; 采用类似的方法, 可制得 $5(\mathrm{NiO}+\mathrm{CuO}) /$ AMSNC 和 $10(\mathrm{NiO}+\mathrm{CuO}) / \mathrm{AMSNC}$ 催化剂。染料的 光催化降解率顺序: $100 \%(10(\mathrm{NiO}+\mathrm{CuO}) / \mathrm{AMSNC})>$ $78.04 \%(5(\mathrm{NiO}+\mathrm{CuO}) / \mathrm{AMSNC})>59.40 \%(1(\mathrm{NiO}+\mathrm{CuO}) /$ $\mathrm{AMSNC})>54.12 \%\left(\mathrm{NH}_{4}-\mathrm{AMSNC}\right)$ 。光催化降解活性 与 $\mathrm{NiO}$ 和 $\mathrm{CuO}$ 半导体的带隙能以及价带与导带位 置密切相关, 利用 $E_{\mathrm{v}}$ 和 $E_{\mathrm{c}}$ 公式分别计算活性 $\mathrm{NiO}$ 和 $\mathrm{CuO}$ 的价带与导带的带隙能 ${ }^{[69-70]}$ 。

$$
\begin{gathered}
E_{\mathrm{v}}=-I=-\chi-0.5 E_{\mathrm{g}} \\
E_{\mathrm{c}}=-A=-\chi+0.5 E_{\mathrm{g}}
\end{gathered}
$$

当负载活性 $\mathrm{NiO}$ 和 $\mathrm{CuO}$ 物种的催化剂暴露于 $\mathrm{UV}$ 光下, 光催化反应随之发生。二种半导体分别吸 收了大于其带隙宽度的光子能量后，电子从 $E_{\mathrm{v}}$ 跃迁 到 $E_{\mathrm{c}}$, 产生光生电子及空穴, 由于 $\mathrm{NiO}$ 和 $\mathrm{CuO}$ 的势 差, 光生电子及空穴发生迁移, 在水溶液中, 吸附 于 $\mathrm{CuO}$ 表面的 $\mathrm{O}_{2}$ 分子与 $E_{\mathrm{c}}$ 上的光生电子作用产生 过氧自由基, 光生空穴与 $\mathrm{H}_{2} \mathrm{O}$ 分子作用生成羟基自 由基和质子, 羟基自由基将吸附于 $\mathrm{CuO}$ 表面的染料 完全氧化 ${ }^{[71]}$ 。

\section{4 结束语}

自上世纪 40 年代以来，人们对碱激发胶凝材料 进行了大量深入的研究, 取得了丰硕的科研成果。 本文从碱激发固体铝硅酸盐胶凝材料的分类、制备 工艺、形成机理以及作为一类新型催化剂材料等诸 多方面, 对该材料的研究进展进行了较为全面深入 的综述, 函待解决的科学问题及工程化问题如下:

1) 碱激发胶凝材料主要采用天然粘土(如: 高 岭土)、各种冶金废渣(如: 粉煤灰、钢渣、矿渣、赤 泥)、各种尾矿(如: 煤矸石, 镍一铜尾矿)等因产地、 造渣工艺之不同、化学成分及矿物相迥然不同，制 
备的碱激发胶凝材料的性能千差万别，还需加大对 该材料的微观结构、激发机理、力学性能、热学性 能和长期耐久性等进行系统地研究, 积累大量数据, 归纳普遍规律, 采取合适的制备工艺, 提高该类材 料的长期耐久性。

2) 要使碱激发胶凝材料尽快满足工程化应用 的要求，建立该材料的技术规范或行业标准刻不容 缓, 因为技术规范或行业标准是设计者, 是施工方 以及用户使用该材料的依据和保障。

3) 鉴于碱激发胶凝材料作为新型结构材料的 工程化应用还有大量工作和漫长的时间等待, 拓宽 碱激发胶凝材料新的研究及应用领域, 尤其是作为 先进催化材料的研究及应用势在必行。

\section{参考文献:}

[1] SALAHUDDIN M B M, NORKHAIRUNNISA M, MUSTAPHA F. A review on thermophysical evaluation of alkali-activated geopolymers. Ceram. Int., 2015, 41: 4273-4281.

[2] RASHAD A M. Alkali-activated metakaolin: a short guide for civil engineer - an overview. Constr. Build. Mater., 2013, 41: 751-765.

[3] KHALE D, CHAUDHARY R. Mechanism of geopolymerization and factors influencing its development: a review. J. Mater. Sci., 2007, 42: 729-746.

[4] PACHECO-TORGAL F, CASTRO-GOMES J, JALALI S. Alkaliactivated binders: a review part 1 . Historical background, terminology, reaction mechanisms and hydration products. Constr. Build. Mater., 2008, 22: 1305-1314.

[5] KOMNITSAS K, ZAHARAKI D. Geopolymerisation: a review and prospects for the minerals industry. Miner. Eng., 2007, 20: $1261-1277$.

[6] ROY D M. Alkali activated cement opportunities and challenges. Cem. Concr. Res., 1999, 29: 249-254.

[7] DUXSON P, FERNANDEZ-JIMENEZ A, PROVIS J L, et al. Geopolymer technology: the current state of the art. J. Mater. Sci., 2007, 42: 2917-2933.

[8] DAVIDOVITS J. Geopolymers and geopolymeric new materials. J. Therm. Anal., 1989, 35: 429-441.

[9] DAVIDOVITS J. Geopolymers: inorganic polymer new materials. J. Therm. Anal., 1991, 37: 1633-1656.

[10] HeitSMANN R F, FitZGERALD M, SAWYER J L. U.S. Patent, No.4643137. 1987.

[11] MALLICOAT S, SARIN P, KRIVEN W M. Novel alkali-bonded ceramic filtration membranes. Ceram. Eng. Sci. Proc., 2008, 26(8): $37-44$.

[12] RAHIER H, VAN MELE B, BIESEMANS $\mathrm{M}$, et al. Low-temperature synthesized aluminosilicate glasses. J. Mater.
Sci., 1996, 31: 71-79.

[13] PURDON A O. The action of alkalis on blast-furnace slag. J. Soc. Chem. Ind., 1940, 59: 191-202.

[14] XU H, VAN DEVENTER J S J. The effect of alkali metals on the formation of geopolymeric gels from alkali-feldspar. Colloids Surf. A, 2003, 216(1/2/3): 27-44.

[15] XU H, VAN DEVENTER J S J. Geopolymerisation of multiple minerals. Miner. Eng., 2002, 15: 1131-1139.

[16] FERNANDEZ-JIMENEZ A, PALOMO A, CRIADO M. Microstructure development of alkali-activated fly ash cement: a descriptive model. Cem. Concr. Res., 2005, 35: 1204-1209.

[17] FERNANDEZ-JIMENEZ A, PALOMO A, SOBRADOS I, et al. The role played by the reactive alumina content in the alkaline activation of fly ashes. Microporous Mesoporous Mater., 2006, 91: $111-119$.

[18] BELLMANN F, STARK J. Activation of blast furnace slag by a new method. Cem. Concr. Res., 2009, 39: 644-650.

[19] CHENG T W, CHIU J P. Fire-resistant geopolymer produced by granulated blast furnace slag. Miner. Eng., 2003, 16: 205-210.

[20] SONG S, SOHN D, JENNINGS H M, et al. Hydration of alkaliactivated ground granulated balst furnace slag. J. Mater. Sci., 2000, 35: 249-257.

[21] ZHANG Y J, ZHAO Y L, LI H H, et al. Structure characterization of hydration products generated by alkaline activation of granulated blast furnace slag. J. Mater. Sci., 2008(43): 7141-7147.

[22] XU H, VAN DEVENTER J S J. The geopolymerisation of aluminosilicate minerals. Int. J. Miner. Process., 2000, 59(3): 247-266.

[23] YIP C K, VAN DEVENTER J S J. Microanalysis of calcium silicate hydrate gel formed within a geopolymeric binder. J. Mater. Sci., 2003, 38: 3851-3860.

[24] YIP C K, LUKEY G C, VAN DEVENTER J S J. Effect of blast furnace slag addition on microstructure and properties of metakaolinite geopolymeric materials. Ceram. Trans., 2004, 153: 187-209.

[25] ZHANG Y J, WANG Y C, XU D L, et al. Mechanical performance and hydration mechanism of geopolymer composite reinforced by resin. Mater. Sci. Eng. A, 527: 2010, 6574-6580.

[26] ZHANG Y J, LI S, WANG Y C, et al. Microstructural and strength evolutions of geopolymer composite reinforced by resin exposed to elevated temperature. J. Non-Crystal. Solids, 2012, 358: 620-624.

[27] ZHANG Y J, LI S, XU D L, et al. A novel method for preparation of organic resins reinforced geopolymer composites. J. Mater. Sci., 2010, 45: 1189-1192.

[28] YIP C K, LUKEY G C, VAN DEVENTER J S J. The coexistence of geopolymeric gel and calcium silicate hydrate at the early stage of alkaline activation. Cem. Concr. Res., 2005, 35: 1688-1697.

[29] GORETTA K C, CHEN N, GUTIERREZ-MORA F, et al. Solid-particle erosion of a geopolymer containing fly ash and 
blast-furnace slag. Wear, 2004, 256: 714-719.

[30] OH J E, MONTEIRO P J M, JUN S S, et al. The evolution of strength and crystalline phases for alkali-activated ground blast furnace slag and fly ash-based geopolymers. Cem. Concr. Res., 2010, 40: 189-196.

[31] KOMNITSAS K, ZAHARAKI D, PERDIKATSIS V. Effect of synthesis parameters on the compressive strength of low-calcium ferronickel slag inorganic polymers. J. Hazard. Mater., 2009, 161: $760-768$.

[32] PAN Z, LI D, YU J, et al. Properties and microstructure of the hardened alkali-activated red mud-slag cementitious material. Cem. Concr. Res., 2003, 33: 1437-1441.

[33] ACHECO-TORGal F, CASTRO-GOMES J, JALALI S. Properties of tungsten mine waste geopolymeric binder. Constr. Build. Mater., 2008, 22: 1201-1211.

[34] RODRIGUEZ E D, BERNAL S A, PROVIS J L, et al. Geopolymers based on spent catalyst residue from a fluid catalytic cracking (FCC) process. Fuel, 2013, 109: 493-502.

[35] SINGH P S, BASTOW T, TRIGG M. Structural studies of geopolymers by 29Si and 27Al MAS-NMR. J. Mater. Sci., 2005, 40: 3951-3961.

[36] DUXSON P, LUKEY G C, VAN DEVENTER J S J. Thermal conductivity of metakaolin geopolymers used as a first approximation for determining gel interconnectivity. Ind. Eng. Chem. Res., 2006, 45(23): 7781-7788.

[37] WANG H, LI H, YAN F. Reduction in wear of metakaolinite-based geopolymer composite through filling of PTFE. Wear, 2005, 258 (10): 1562-1566.

[38] NAIR B G, ZHAO Q, COOPER R F. Geopolymer matrices with improved hydrothermal corrosion resistance for high-temperature applications. J. Mater. Sci., 2007, 42: 3083-3091.

[39] BELL J L, GORDON M, KRIVEN W M. Use of geopolymeric cements as a refractory adhesive for metal and ceramic joins. Ceram. Eng. Sci. Proc., 2005, 26(3): 407-413.

[40] GARCIA-LODEIRO I, PALOMO A, FERNANDEZ-JIMENEZ A. Alkali-aggregate reaction in activated fly ash systems. Cem. Concr. Res., 2007, 37(2): 175-183.

[41] BAKHAREV T, SANJAYAN J, CHENG Y B. Resistance of alkaliactivated slag concrete to acid attack. Cem. Concr. Res., 2003, 33: 1607-1611.

[42] SHI C, STEGEMANN J. Acid corrosion resistance of different cementing materials. Cem. Concr. Res., 2000, 30: 803-808.

[43] BAKHAREV T, SANJAYAN J G, CHENG Y B. Sulfate attack on alkali-activated slag concrete. Cem. Concr. Res., 2002, 32: 211-216.

[44] PALOMO A, BLANCO-VARELA M T, GRANIZO M L, et al. Chemical stability of cementitious materials based on metakaolin.
Cem. Concr. Res., 1999, 29(7): 997-1004.

[45] ROY D M, JIANG W, SILSBEE M. Chloride diffusion in ordinary, blended, and alkali-activated cement pastes and its relation to other properties. Cem. Concr. Res., 2000, 30: 1879-1884.

[46] PUERTAS F, AMAT T, FERNANDEZ-JIMENEZ A, et al. Mechanical and durable behaviour of alkaline cement mortars reinforced with polypropylene fibres. Cem. Concr. Res., 2003, 33: 2031-2036.

[47] GLASSER FP. Progress in the immobilization of radioactive wastes in cement, Cem. Concr. Res., 1992, 22: 201-216.

[48] VAN JAARSVELD J G S, VAN DEVENTER J S J, SCHWARTZMAN A. The potential use of geopolymeric materials to immobilize toxic metals: part II. Material and leaching characteristics. Miner. Eng., 1999, 12(1): 75-91.

[49] QIAN G, SUN D D, TAY J H. Immobilization of mercury and zinc in an alkali-activated slag matrix. J. Hazard. Mater., 2003, 101(1): $65-77$.

[50] JAN D. Immobilization of $\mathrm{Cr}^{6+}, \mathrm{Cd}^{2+}, \mathrm{Zn}^{2+}$ and $\mathrm{Pb}^{2+}$ in alkaliactivated slag binders. Cem. Concr. Res., 2002, 32(12): 1971-1979.

[51] SAZAMA P, BORTNOVSKY O, DEDECEK J, et al. Geopolymer based catalysts-new group of catalytic materials. Catal. Today, 2011, 164: 92-99.

[52] CASCA-TIRADO J R, MANZANO-RAMIREZ A, VILLASENORMORA C, et al. Incorporation of photoactive $\mathrm{TiO}_{2}$ in an aluminosilicate inorganic polymer by ion exchange. Microporous Mesoporous Mater., 2012, 153: 282-287.

[53] CASCA-TIRADO J R, MANZANO-RAMIREZ A, VAZQUEZLANDAVERDE $\mathrm{P}$ A, et al. Ion-exchanged geopolymer for photocatalytic degradation of a volatile organic compound. Mater. Lett., 2014, 134: 222-224.

[54] CANDAMANO S, FRONTERA P, MACARIO A, et al. Preparation and characterization of active $\mathrm{Ni}$-supported catalyst for syngas production. Chem. Eng. Res. Des., 2015, 96: 78-86.

[55] SHARMA S, MEDPELLI D, CHEN S, et al. Calcium-modified hierarchically porous aluminosilicate geopolymer as a highly efficient regenerable catalyst for biodiesel production. RSC Adv., 2015, 5: 65454-65461.

[56] ZHANG Y J, LIU L C, XU Y, et al. A new alkali-activated steel slag-based cementitious material for photocatalytic degradation of organic pollutant from waste water. J. Hazard. Mater., 2012, 209-210: $146-150$

[57] KANG L, ZHANG Y J, WANG L L, et al. Alkali-activated steel slag-based mesoporous material as a new photocatalyst for degradation of dye from wastewater. Integr. Ferroelectr., 2015, 162: $8-17$.

[58] AHMARUZZAMAN M. A review on the utilization of fly ash. 
Prog. Energy Combust. Sci., 2010, 36 (3): 327-363.

[59] LI L, WANG S, ZHU Z. Geopolymeric adsorbents from fly ash for dye removal from aqueous solution. J. Colloid Interface Sci., 2006, 300 (1): 52-59.

[60] AL-ZBOONA K, AL-HARAHSHEH M S, HANI F B. Fly ash-based geopolymer for $\mathrm{Pb}$ removal from aqueous solution. $J$. Hazard. Mater., 2011, 188: 414-421.

[61] WANG S, LI L, ZHU Z H. Solid-state conversion of fly ash to effective adsorbents for $\mathrm{Cu}$ removal from wastewater. J. Hazard. Mater., 2007, 139(2): 254-259.

[62] HUANG Y, HAN M. The influence of $\alpha-\mathrm{Al}_{2} \mathrm{O}_{3}$ addition on microstructure, mechanical and formaldehyde adsorption properties of fly ash-based geopolymer products. J. Hazard. Mater., 2011, 193: 90-94.

[63] CHENG T W, LEE M L, KO M S, et al. The heavy metal adsorption characteristics on metakaolin-based geopolymer. Appl. Clay Sci., 2012, 56: 90-96.

[64] ZHANG Y J, LIU L C. Fly ash-based geopolymer as a novel photocatalyst for degradation of dye from wastewater. Particuology, 2013, 11: 353-358.

[65] ZHANG Y J, LIU L C, NI, L L, et al. A facile and low-cost synthesis of granulated blast furnace slag-based cementitious material coupled with $\mathrm{Fe}_{2} \mathrm{O}_{3}$ catalyst for treatment of dye wastewater. Appl. Catal. B, 2013, 138-139: 9-16.

[66] ZHANG Y J, CHAI Q. Alkali-activated blast furnace slag-based nanomaterial as a novel catalyst for synthesis of hydrogen fuel. Fuel, 2014, 115: 84-87.

[67] ZHANG Y J, KANG L, LIU L C, et al. Synthesis of a novel alkali-activated magnesium slag-based nanostructural composite and its photocatalytic performance. Appl. Surf. Sci., 2015, 331: 399-406.

[68] ZHANG Y J, KANG L, SI H X, et al. A novel alkali-activated magnesium slag based nanocomposite for photocatalytic production of hydrogen. Integr. Ferroelectr., 2014, 154: 120-127.

[69] BUTLER M A, GINLEY D S. Prediction of flatband potentials at semiconductorelectrolyte interfaces from atomic electronegativities, J. Electrochem. Soc., 1978, 125: 228-232.

[70] XU Y, SCHOONEN M A A. The absolute energy positions of conduction and valence bands of selected semiconducting minerals. Am. Mineral., 2000, 85: 543-556.

[71] ZHANG Y J, KANG L, LIU L C. Alkali-activated Cements for Photocatalytic Degradation of Organic Dyes. in: PACHECOTORGAL F, LABRINCHA J A, Leonelli LEONELLI C, et al. Handbook of Alkali-activated Cements, Mortars and Concretes, UK: Woodhead Publishing, 2015: 729-777. 